\title{
Physical Mathematics and the Fine-Structure Constant
}

\author{
Michael A. Sherbon \\ Case Western Reserve University Alumnus \\ E-mail:michael.sherbon@case.edu
}

July 11,2018

\begin{abstract}
Research into ancient physical structures, some having been known as the seven wonders of the ancient world, inspired new developments in the early history of mathematics. At the other end of this spectrum of inquiry the research is concerned with the minimum of observations from physical data as exemplified by Eddington's Principle. Current discussions of the interplay between physics and mathematics revive some of this early history of mathematics and offer insight into the fine-structure constant. Arthur Eddington's work leads to a new calculation of the inverse fine-structure constant giving the same approximate value as ancient geometry combined with the golden ratio structure of the hydrogen atom. The hyperbolic function suggested by Alfred Landé leads to another result, involving the Laplace limit of Kepler's equation, with the same approximate value and related to the aforementioned results. The accuracy of these results are consistent with the standard reference. Relationships between the four fundamental coupling constants are also found.
\end{abstract}

Keywords fine-structure constant, dimensionless physical constants, history of mathematics, golden ratio, history of physics, mathematical constants, fundamental constants.

\section{Introduction}

Natalie Paquette has stated that "Mathematicians discovered group theory long before physicists began using it. In the case of string theory, it is often the other way around. Physics has lent the dignity of its ideas to mathematics. The result is what Greg Moore has called physical mathematics." [1, 2]. For an additional comprehensive overview of these results see the work of Terry Gannon [3]. Édouard Brézin is "... in agreement with Paquette. We should expect to see more new insights in mathematics emerging from the rich structure of physical problems." [4]. Robbert Dijkgraaf states, "Mathematics has a long history of drawing inspiration from the physical 
sciences, going back to astrology, architecture and land measurements in Babylonian and Egyptian times." [5]. From Arnold Sommerfeld in the history of physics [6], Stephen Brush writes that in 1916 "Arnold Sommerfeld generalized Bohr's model in to include elliptical orbits in three dimensions. He treated the problem relativistically (using Einstein's formula for the increase of mass with velocity), ... According to historian Max Jammer, this success of Sommerfeld's finestructure formula ' ... served also as an indirect confirmation of Einstein's relativistic formula for the velocity dependence of inertia mass." [7]. The electromagnetic coupling constant determining the strength of its interaction is the fine-structure constant $\alpha=e^{2} / \hbar c$ in $\operatorname{cgs}$ units with the elementary charge $e$, the reduced Planck's constant $\hbar=h / 2 \pi$ and the speed of light $c$. As Max Born wrote, "It is clear that the explanation of this number must be the central problem of natural philosophy." [8]. This was a view also shared by Wolfgang Pauli [9, 10].

\section{Eddington's Principle}

Regarding relativity and quantum mechanics in Dirac's work on the electron spin, "It was not till the initiative inspired by Dirac's equation that Eddington had the notion of a bridge between the theories. Comparing treatments would give numerical values for certain physical constants." [11]. Helge Kragh writes, "Like many contemporary physicists, Dirac believed that ultimately $\alpha$ should be explainable by physical theory. As late as 1978, he wrote: 'The problem of explaining this number [fine-structure constant] is still completely unsolved. ... I think it is perhaps the most fundamental unsolved problem of physics at the present time, and I doubt very much whether any really big progress will be made in understanding the fundamentals of physics until it is solved." [12].

Helge Kragh also states that, "By 1929 the fine-structure constant was far from new, but it was only with Eddington's work that the dimensionless combination of constants of nature was elevated from an empirical quantity appearing in spectroscopy to a truly fundamental constant [12]. In addition, Kragh states, "He was also the first to argue that $\alpha$ was of deep cosmological significance and that it should be derivable from fundamental theory." [13]. Eddington asked himself what the minimum amount of data from observation was required for a physical theory. This led to Eddington's Principle from which he maintained that the value of the inverse finestructure constant was 136, which he later changed to 137. Eddington's Principle is defined as: "All the quantitative propositions of physics, that is, the exact values of the pure numbers that are constants of science, may be deduced by logical reasoning from qualitative assertions without making any use of quantitative data derived from observation." [14].

\section{Fine-structure constant}

Nikos Salingaros says, "Eddington anticipated results of current interest. He discovered the Majorana spinors, and was responsible for the standard $\gamma^{5}$ notation as well as the notion of chirality. Furthermore, Eddington defined Clifford algebras in eight and nine dimensions which are now appearing in grand unified gauge and supersymmetric theories. A point which Eddington cleared up, yet is still misunderstood, is that the Dirac algebra corresponds to a five-dimensional base space." [15]. In addition, "Eddington did not clearly anticipate current physical supersymmetry 
theories. He did sense that a larger Clifford algebra would be useful in a symmetrical description of nature, and in this aspect he was entirely correct." [15], also see [16]-[19]. As the use of advanced algebras grew in the study of symmetry another development of interest happened in 1974 when Howard Georgi and Sheldon Glashow proposed SU(5) as " ... the gauge group of the world - that all elementary particle forces (strong, weak, and electromagnetic) are different manifestations of the same fundamental interaction involving a single coupling strength, the fine-structure constant [20]. As Giora Shaviv points out “... if you consider a symmetric matrix in $16=4 \times 4$ dimensions, then the number of independent terms in this matrix is ... 136." [21]. Eddington: $16+\left(16^{2}-16\right) / 2=256-120=136$. Inverse fine-structure constant is a root of:

$$
x^{4}-136 x^{3}-136 x^{2}-818 x+1=0 .
$$

This equation gives a value of for $x$ as $\alpha^{-1} \simeq 137.035999168$. The inferred value determined from quantum electrodynamic theory and experiment with the least standard uncertainty in CODATA results is $\alpha^{-1} \simeq 137.035999160$ (33) [22]. The other root of the equation is approximately $1 / 818$ and $818=(136+1 / 3) 6=(4 \times 136)+(2 \times 137)$.

In William Eisen's research on the geometry of the "Golden Apex of the Great Pyramid," his interpretation involving Euler's identity, $\exp (i \pi)+1=0$ shows a drawing of four curves of $e^{x}$ from $x=0$ to $x=\pi$, one curve on each side and labelled the "Graphical Representation of the Exponential Function to the Base $e$." [23]. Dividing the side lengths of the Great Pyramid by $\pi$ lengths results in a small central square called the Golden Apex, the geometry associated with the fivefold symmetry of the Great Pyramid and the four forces of nature [24]. Golden Apex A:

$$
A=e^{\pi}-7 \pi-1 \simeq \sqrt{2} / 3 \pi \simeq \phi^{2} / 2 K \simeq 3 / 20 .
$$

$A$ is the side length of the Golden Apex square. $\sqrt{A} \simeq e / 7$ and $A+1=e^{\pi}-7 \pi \simeq R$, radius of the regular heptagon with side equal to one, $R^{-1}=2 \sin (\pi / 7)$. The $\sin (2 \pi A) \simeq \phi / 2$, where $\phi$ is the golden ratio [24]. Also related, $\sqrt{A}+1 \simeq K / 2 \pi$, see references in [24]. The polygon circumscribing constant is $K \simeq 2 \tan (3 \pi / 7) \simeq \phi^{2} / 2 A$ [25]. The Golden Apex $A$ and the golden ratio relate simple approximations involving the four fundamental coupling constants:

$$
A \simeq \sqrt{\phi / 3} / \ln \alpha^{-1} \simeq 2 \sqrt{3 \phi} \alpha_{w} \simeq \alpha_{s} \sqrt{\phi} \simeq 7 \sqrt{3 \phi} / \ln \alpha_{G}^{-1},
$$

where $\alpha_{w}$ is the weak nuclear force coupling constant, $\alpha_{s}$ is the strong nuclear force coupling constant and $\alpha_{G}$ is the gravitational coupling constant [26]. The inclusion of the four fundamenal coupling constants in the Golden Apex design is part of the symbolic interpretation presented by William Eisen [23]. Other brief approximations involving the four fundamental coupling constants include: $A \simeq 4 \pi \phi \alpha \simeq \alpha_{w} / \sin ^{2} \theta_{w} \simeq \pi \phi \alpha_{s} / 4 \simeq K \sqrt{\pi} / \ln \alpha_{G}^{-1}$. The Weinberg angle, $\sin ^{2} \theta_{w} \simeq \cot (3 \pi / 7) \simeq \sqrt{7 \alpha}$. The Golden Apex $A$ has more relatively simple approximations:

$$
A \simeq 7 \sqrt{\alpha} / 4 \simeq \lambda \sqrt{7 \alpha} \simeq 7 \alpha \sqrt{K} \simeq 7 \sqrt{\alpha / \phi} / \pi
$$

with the fine-structure constant and the related Laplace limit of Kepler's equation, $\lambda$ [27, 28]. Raji Heyrovska found that the Bohr radius was divided by the golden ratio into two different sections giving $\alpha^{-1} \simeq\left(360 / \phi^{2}\right)-\left(2 / \phi^{3}\right)$ [29], with a difference from experiment possibly due to the g-factors of the electron and proton [30]. Her equation was then extended with the 
Golden Apex $A$ and the polygon circumscribing constant $K$ [31]. Related to the Golden Apex geometry [27], $667-178-49=440,4+49+49+178=280,667+136-137=666$ and $4+49+667=280+440=720$ [24]. Fine-structure constant $\alpha \simeq A / x$, where $x$ is a root of:

$$
4 x^{3}-49 x^{2}-667 x-178=0 .
$$

This equation gives a value for $\alpha^{-1} \simeq 137.035999168$. Another approximation involving the golden ratio is also related to Eq.(1), recalling some of Eddington's work, $136+136+48=$ $440-120$ and $280=136+144$. Also, $346-280-36=30,280-30=250,3 \times 36=108$, $3 \times 48=144$ and $108^{2}+144^{2}=180^{2}$ [24]. This polynomial equation gives a value for the inverse fine-structure constant $\alpha^{-1} \simeq 137.035999168$ and $\alpha^{-1} \simeq \phi x$, where $x$ is a root of:

$$
3 x^{4}-250 x^{3}-346 x^{2}+48 x-36=0 .
$$

In 1939 Alfred Landé, who found the Landé g-factor of the electron, stated that the $\sinh (2 \pi)$ was significant to an understanding of the fine-structure constant [32, 33]. From the Golden Apex view, $\alpha^{-1} \simeq \lambda \sinh (2 \pi) / K A \simeq \sinh (2 \pi) / \lambda \sqrt{K}$ and $\alpha \sinh (2 \pi) \simeq \tan ^{-1}(4 / \pi) / \tan ^{-1}(1 / 2)$, base angle of the physical structure defining the Golden Apex $A$ divided by the ancient 'golden angle' [24, 31]. Another Landé inspired approximation of $\alpha \sinh (2 \pi)$ is a root of a cubic equation. The root of this cubic equation divided into $\sinh (2 \pi)$ gives a value for $\alpha^{-1} \simeq 137.035999168$.

$$
33 x^{3}-122 x^{2}+377 x-517=0,
$$

with Fibonacci numbers, $517-377=140,377-137=240,720-377=377-34$ and $89=$ $122-33$ [24]. Also, $818-440-377=137-136$. Related to Alfred Landé again is the Laplace limit of Kepler's equation, $\lambda \simeq A / \sqrt{7 \alpha} \simeq \phi \pi \sqrt{A / K}[28]$ and $\pi / \phi^{2} \simeq \sqrt{1+\lambda^{2}}$ where $\sqrt{1+\lambda^{2}}=\mu$ is the real fixed point of the hyperbolic cotangent related to the anomalous magnetic moment of the electron: $\sinh \mu \simeq \lambda^{-1}$ and $\alpha \sinh (2 \pi) \simeq 2 \mu \sqrt{\lambda}$ [34]. For the value of $\alpha^{-1} \simeq 137.035999168$ as the inverse fine-structure constant: $\alpha^{-1} \simeq x / \lambda$, where $\mathrm{x}$ is a root of:

$$
5 x^{3}-455 x^{2}+86 x-375=0 .
$$

Related, $455-375=360-280,455 / 5=5+86,375-136-86=153$ and $153 / 136=9 / 8$, from the source geometry of the physical structure determining the Golden Apex [27]. Additionally, $137+137=360-86$, and $455-375=440-360$. Also, $360+360 / 5=432 \simeq 360 \pi / \phi^{2} \simeq$ $\phi \sinh (2 \pi)$. With hyperbolic functions, $\phi^{-1} \simeq \sinh ^{-1} \lambda \simeq \cosh ^{-1} \mu$ and $\phi \simeq \sinh ^{-1}(2 \mu)$. The dimensionless proton-electron mass ratio is $m_{p} / m_{e} \simeq \phi^{4} \sinh (2 \pi)$ and $\phi^{4} \simeq A+A^{-1} \simeq 7-A$.

\section{Squaring the circle}

Squaring the circle has been a metaphor for the effort to unify relativity and quantum theory [35]. Related to the squaring of the circle are more approximations involving the four fundamental coupling constants. The proportion significant to 'squaring the circle' in the classical tradition was found by John Michell and presented in his study of what he named the Cosmological Circle: $3 / 11 \simeq \sqrt{\phi}-1 \simeq \sqrt{\phi} \alpha / \alpha_{w} \simeq \alpha_{s} \sqrt{\phi S} \simeq \sqrt{\phi} / \ln \left(\ln \alpha_{G}^{-1}\right)[31] . S$ is the silver constant from the 
regular heptagon [34]. $S=4 \sin ^{2}(2 \pi / 7)=2+2 \sin (2 \pi / 7)=2+\sqrt{S} / R$, the regular heptagon and the golden ratio are both closely associated with the classical geometry of 'squaring the circle.' $S \simeq \sqrt{\pi / 2 A} \simeq 4 \sqrt{\lambda} \simeq 2 \phi$ and $S^{-1} \simeq \tanh \pi^{-1}$. Also, $3 / 11=120 / 440 \simeq \sqrt{A / 2} \simeq$ $A \sqrt{S} \simeq \sqrt{\alpha} / 2 A \simeq \sqrt{\phi A / S} \simeq 76.3 / 280$ and the apex angle is $76.3^{\circ}$ [24]. The Golden Apex $A \simeq \pi /(3 \times 7) \simeq 2 \pi \alpha S$. [28, 31]. The conceptual structure defining the Golden Apex has a significant relationship to advanced algebras, modular forms and fundamental physics [27].

\section{Conclusion}

These calculations of the inverse fine-structure constant are aimed toward a fuller explanation of the fundamental physics and the interrelated mathematics. As Albert Einstein noted, "Our experience up to date justifies us in feeling sure that in Nature is actualized the ideal of mathematical simplicity. It is my conviction that pure mathematical construction enables us to discover the concepts and the laws connecting them which give us the key to the understanding of the phenomena of Nature." [36]. Besides Eddington, Einstein's view in this regard was also shared most notably by Paul Dirac [37]. Natalie Paquette, quoted in the introduction, concludes her article with, "If mathematics and physics are in so many respects in equipoise, then the differences between them may be less a matter of their content than their technique; and that, in the end, they serve to show that there is only one reality to which they both appeal." [1].

\section{Acknowledgments}

Special thanks to Case Western Reserve University, MathWorld and WolframAlpha.

\section{References}

[1] Paquette, N. "A View from the Bridge: On the relationship between mathematics and physics," Inference: International Review of Science, 3, 4 (2018). inference-review-bridge.

[2] Moore, G.W. "Physical Mathematics and the Future," Vision talk, Strings conference, Princeton, 10 (2014). gmoore.pdf.

[3] Gannon, T. "Moonshine beyond the Monster: The bridge connecting algebra, modular forms and physics," Cambridge: Cambridge University Press, 2006.

[4] Brézin, E. "Physical Mathematics," Inference: International Review of Science, 4, 1 (2018). inference-physical-mathematics.

[5] Dijkgraaf, R. "Mathematical Structures," in Henneaux, M., Alexander, S. \& Gross, D.J. eds. Quantum Structure Of Space And Time, Singapore: World Scientific, 2007.

[6] Eckert, M. Arnold Sommerfeld: Science, Life and Turbulent Times 1868-1951, New York, NY: Springer, 2013, xi.

[7] Brush, S.G. Making 20th Century Science: How Theories Became Knowledge, Oxford, UK: Oxford University Press, 2015, 220. 
[8] Born, M. "The Mysterious Number 137," Proceedings of the Indian Academy of Science A 6, 533-561 (1935). ias.ac.in-born.

[9] Miller, A.I. Deciphering the Cosmic Number, New York, NY: W.W. Norton, 2009.

[10] Sherbon, M.A. "Wolfgang Pauli and the Fine-Structure Constant," Journal of Science, 2, 3, 148-154 (2012). hal-01304518.

[11] Kilmister, C.W. Eddington's Search for a Fundamental Theory, Cambridge: Cambridge University Press, 1994, 4.

[12] Kragh, H. "Magic Number: A Partial History of the Fine-Structure Constant," Archive for History of Exact Sciences, 57, 5, 395-431 (2003). s00407-002-0065-7.

[13] Kragh, H. “On Arthur Eddington's Theory of Everything,” (2015). arXiv:1510.04046.

[14] Whittaker, E. "Eddington's Principle in the Philosophy of Science," in Heine, V., Rees, M.J. et al, A.S. Eddington and the Unity of Knowledge, Cambridge: Cambridge University Press, 2013.

[15] Salingaros, N. "Some Remarks on the Algebra of Eddington's E numbers," Foundations of Physics, 15, 6, 683-691 (1985). doi/10.1007/BF00738296.

[16] Eddington, A.S. Fundamental Theory, Cambridge: Cambridge University Press, 1946.

[17] Bekenstein, J.D. "The Fine-Structure Constant: From Eddington's Time to Our Own," in Ullmann-Margalit, E. ed. The Prism of Science, New York, NY: Springer, 1986. 209-224.

[18] Durham, I.T. "Sir Arthur Eddington and the Foundations of Modern Physics," (2006). arXiv:quant-ph/0603146.

[19] Rickles, D. "All Possible Perspectives: A (Partial) Defence of Eddington's Physics," in Durham, I.T. \& Rickles, D. eds. Information and Interaction: Eddington, Wheeler and the Limits of Knowledge, New York, NY: Springer, 2016.

[20] Georgi, H. \& Glashow, S.L. "Unity of All Elementary Particle Forces," Physical Review Letters, 32, 8, 438 (1974), in Froggatt, C.D. \& Nielsen, H.B. eds. Origin of Symmetries, Singapore: World Scientific, 1991. doi/10.1103/PhysRevLett.32.438.

[21] Shaviv, G. The Synthesis of the Elements: The Astrophysical Quest for Nucleosynthesis and What It Can Tell Us About the Universe, New York, NY: Springer, 2012, 156.

[22] Mohr, P.J., Newell, D.B. \& Taylor, B.N. "CODATA Recommended Values of the Fundamental Physical Constants: 2014," Reviews of Modern Physics, 88, 035009 (2016). doi/10.1103/RevModPhys.88.035009

[23] Eisen, W. "The Mysteries of the Constants (e) and (i)," in The Universal Language of Cabalah, Marina Del Rey, CA: DeVorss, 1989, 160-178. 
[24] Sherbon, M.A. "Quintessential Nature of the Fine-Structure Constant," Global Journal of Science Frontier Research A, 15, 4, 23-26 (2015). hal-01174786.

[25] Sloane, N.J.A. "Polygon Circumscribing Constant," Sequence A051762 in The On-Line Encyclopedia of Integer Sequences. oeis.org/A051762.

[26] Huang, K. Fundamental Forces of Nature, Singapore: World Scientific, 2007.

[27] Sherbon, M.A. "Fundamental Physics and the Fine-Structure Constant," International Journal of Physical Research, 5, 2, 46-48 (2017). hal-01312695.

[28] Weisstein, E.W. "Laplace Limit," MathWorld-A Wolfram Web Resource. mathworld.wolfram.com/LaplaceLimit.

[29] Heyrovska, R. "The Golden Ratio, Ionic and Atomic Radii and Bond Lengths," Molecular Physics, 103, 877 - 882 (2005). doi/10.1080/00268970412331333591.

[30] Heyrovska, R. "Golden Ratio Based Fine Structure Constant and Rydberg Constant for Hydrogen Spectra," International Journal of Sciences, 2, 5, 28-31 (2013). ijsciences.com/pub/article/185.

[31] Sherbon, M.A. "Fine-Structure Constant from Golden Ratio Geometry," International Journal of Mathematics and Physical Sciences Research, 5, 2, 89-100 (2018). hal01756341

[32] Landé, A. "Sommerfeld's Fine Structure Constant and Born's Reciprocity," Physical Review, 56, 5, 484 (1939). doi/10.1103/PhysRev.56.482.2.

[33] Landé, A. "The Structure of Electric Particles and the Number 137," Physical Review, 56, 5 (1939) in Barut, A.O. \& Merwe, A. Selected Scientific Papers of Alfred Landé. New York, NY: Springer, 1988, 328. doi/10.1103/PhysRev.56.486.

[34] Sherbon, M.A. "Fundamental Nature of the Fine-Structure Constant," International Journal of Physical Research, 2, 1, 1-9 (2014). hal-01304522.

[35] Hagar, A. "Squaring the Circle: Gleb Wataghin and the prehistory of quantum gravity," Studies in History and Philosophy of Science Part B: Studies in History and Philosophy of Modern Physics, 46, 217-227 (2014). doi/10.1016/j.shpsb.2013.07.005.

[36] Einstein, A. "On the Method of Theoretical Physics," Philosophy of Science, 1, 2, 163-169 (1934). doi/10.1086/286316.

[37] Dirac, P.A.M. "The Relation between Mathematics and Physics," Proceedings of the Royal Society, 59, 2, 122-129 (1940). doi/10.1017/S0370164600012207. 